\title{
Negative Differential Resistance and Super-Poissonian Shot Noise in a System of Single Electron Transistors
}

\author{
G. MichaŁeK and B.R. BuŁKa \\ Institute of Molecular Physics, Polish Academy of Sciences \\ Smoluchowskiego 17, 60-179 Poznań, Poland
}

\begin{abstract}
Currents and their fluctuations in two capacitively coupled single electron transistors were studied within the sequential tunneling approach. A special attention was focused on the effect of the negative differential resistance, which appears due to the Coulomb interactions of accumulated charges on both the single electron transistors. In this case large polarization fluctuations are activated, which results in a significant enhancement of the current shot noise.
\end{abstract}

PACS numbers: 73.23.Hk, 73.40.Gk, 73.50.Td

Recent progress of modern nanotechnology allows the observation of several effects associated with single electron charging of small tunnel structures. A special kind of tunnel nanojunction is the double-barrier junction with a small central electrode. The electrical circuit consists of two such structures demonstrating negative differential resistance (NDR), which Heij et al. recently measured [1]. In addition, in molecular-scale systems Chen et al. also found such effect [2]. The interest in NDR devices results from numerous potential applications, including amplification, high-frequency oscillators, mixers, multipliers, logic, memory, and analog-to-digital converter circuits.

A very important parameter of the electrical circuits is signal-to-noise ratio. The noise analysis gives us additional information about electronic structure, transport properties, and electron-electron interactions. Besides a thermal noise, in electrical circuits there appears a shot noise. Recently, in mesoscopic systems, the shot noise was intensively studied both theoretically and experimentally. It was shown that the current noise could be reduced below the Poissonian value $S_{\text {Poisson }}=2 e I$ due to correlation between conducting electrons. Experimentally, in the single electron transistor (SET), Birk et al. confirmed this reduction (up

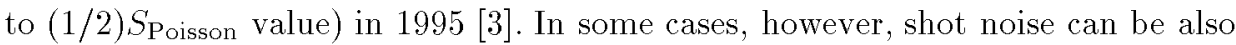
super-Poissonian. Iannaccone et al. found in a resonant tunneling diode an enhancement of the shot noise in the region of NDR [4]. 
To our better knowledge, there is no either theoretical or experimental work on the shot noise in the molecular-scale systems. In this paper, we study the negative differential resistance phenomenon and the shot noises in such system. We perform detailed calculation to find processes responsible for an enhancement of shot noises.

In the work, we describe a sequential tunneling in capacitively coupled non-magnetic SETs (Fig. 1) with discrete electronic structure on the quantum dots. We restrict our consideration to the case where the spacer particles are atomic-size and each of them has only one single electronic level available for the tunneling processes.

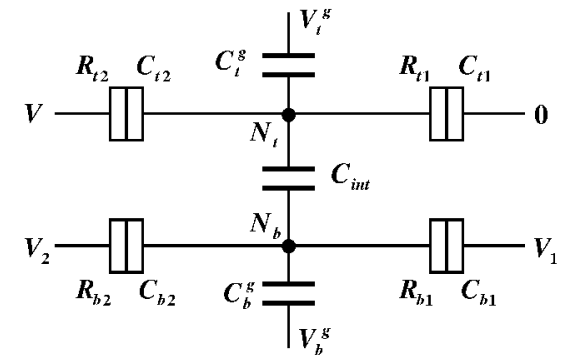

Fig. 1. Electrical scheme of the circuit consisting of two single-electron transistors connected in parallel.

Tunneling process for an electron in the top $(\alpha=\mathrm{t})$ and the bottom ( $\alpha=\mathrm{b}$ ) SET through the left $(i=1)$ and the right $(i=2)$ junction is described by the net tunneling rates $\gamma_{\alpha i}$, which are assumed to be small $\hbar \gamma_{\alpha i} \ll k_{\mathrm{B}} T$. This relation implies that the corresponding tunnel resistances $R_{\alpha i}$ are much larger than the quantum resistance $R_{\mathrm{Q}}=h / 2 e^{2}$ and the electronic transport is dominated by incoherent, sequential tunneling processes [5].

The rates of electron tunneling to $(+)$ and off $(-)$ the $\alpha$ quantum dot through the $i$-th junction, are given by

$$
\Gamma_{\alpha i}^{ \pm}\left(N_{\mathrm{t}}, N_{\mathrm{b}}\right)=\gamma_{\alpha i}\left[1+\exp \left( \pm \frac{E_{\alpha}^{0}-e V_{\alpha i}\left(N_{\mathrm{t}}, N_{\mathrm{b}}\right) \mp E_{\alpha}^{\mathrm{c}}-E_{\mathrm{F}}}{k_{\mathrm{B}} T}\right)\right]^{-1},
$$

where $N_{\mathrm{t}}\left(N_{\mathrm{b}}\right)$ is a charge accumulated on the top (bottom) SET, $E_{\mathrm{F}}$ denotes the Fermi energy and $E_{\alpha}^{c}$ - a charging energy of $\alpha$-th quantum dot (SET), while $-e$ and $T$ stand for an electron charge and temperature, respectively. The voltage drop on the $i$-th junction $\left(V_{\alpha i}\right)$ is given from the Kirchhoff law.

In the stationary state, electric currents can be calculated from the formula

$$
I_{\alpha i}=-e \sum_{N_{\mathrm{t}}, N_{\mathrm{b}}}(-1)^{i}\left[\Gamma_{\alpha i}^{+}\left(N_{\mathrm{t}}, N_{\mathrm{b}}\right)-\Gamma_{\alpha i}^{-}\left(N_{\mathrm{t}}, N_{\mathrm{b}}\right)\right] p_{0}\left(N_{\mathrm{t}}, N_{\mathrm{b}}\right),
$$

where the probability $p_{0}\left(N_{\mathrm{t}}, N_{\mathrm{b}}\right)$ is determined from the relevant master equation, $0=\widehat{M} \widehat{p_{0}}$ (in which the matrix $\widehat{M}$ describes tunneling processes).

To analyze fluctuations in the system we extend the generation-recombination approach [6] for multielectron channels by generalization of the method developed for spinless electrons in a SET [7]. 

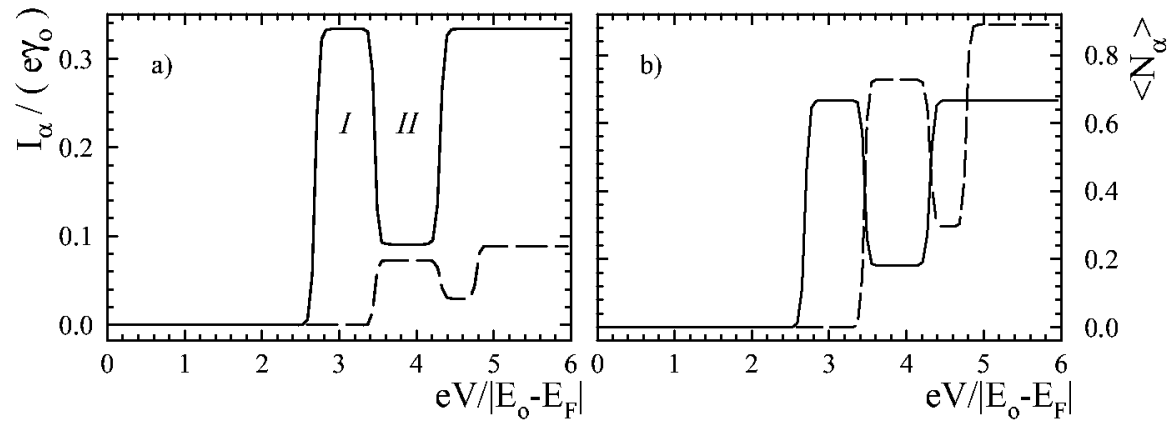

Fig. 2. Current through the top (solid line) and the bottom (dashed line) SET's (a), charge accumulation (b). The parameters are: $C_{\mathrm{t} 1}=0.4 \mathrm{aF}, C_{\mathrm{t} 2}=0.2 \mathrm{aF}, C_{\mathrm{b} 1}=$ $0.15 \mathrm{aF}, C_{\mathrm{b} 2}=0.25 \mathrm{aF}, C_{\mathrm{int}}=0.5 \mathrm{aF}, C_{\alpha}^{\mathrm{g}}=0, E_{\mathrm{t}}^{0}=0.5\left|E_{0}-E_{\mathrm{F}}\right|, E_{\mathrm{b}}^{0}=\left|E_{0}-E_{\mathrm{F}}\right|$, $k_{\mathrm{B}} T=0.01\left|E_{0}-E_{\mathrm{F}}\right|, \gamma_{\mathrm{t} 1}=\gamma_{0}, \gamma_{\mathrm{t} 2}=0.5 \gamma_{0}, \gamma_{\mathrm{b} 1}=0.8 \gamma_{0}, \gamma_{\mathrm{b} 2}=0.1 \gamma_{0}, V_{\alpha}^{\mathrm{g}}=V_{1}=0$ and $V_{2}=V . \gamma_{0}$ is taken as a unity in our calculations.

To characterize system in the stationary state we have calculated numerically the bias voltage dependence of the current $I_{\mathrm{t}}=I_{\mathrm{t} 1}=I_{\mathrm{t} 2}\left(I_{\mathrm{b}}=I_{\mathrm{b} 1}=I_{\mathrm{b} 2}\right)$ flowing through the top (bottom) SET respectively and the average charge accumulated on each quantum dot $\left\langle n_{\alpha}\right\rangle$. The results are presented in Fig. 2a,b. Due to the Coulomb blockade effect, the currents can flow through the system only above some threshold voltages, which are different for both SETs. This difference is the result of the various capacitance $C_{\alpha i}$ of each tunnel junction, the different single electron charging energy $E_{\alpha}^{c}$ and the energy level $E_{\alpha}^{0}$. Each curve in Fig. 2a,b has steps which result from opening new electron channels. These steps are clearly seen only for $k_{\mathrm{B}} T \ll E_{\mathrm{t}}^{\mathrm{c}}, E_{\mathrm{b}}^{\mathrm{c}}$, while for higher $T$ they are smeared out. The amplitude of the tunneling currents depends on tunneling rates $\gamma_{\alpha i}$. When the voltage $V_{2}$ exceeds the threshold voltage $V_{\mathrm{b}}^{\text {th }}$, then current begins to flow through the bottom island. It may lead to quite large (when $\gamma_{\mathrm{b} 2} \ll \gamma_{\mathrm{b} 1}$ ) accumulation of the charge on the bottom quantum dot. The charge accumulated on the bottom transistor blocks the current channel through the top SET. It makes some reduction of the current $I_{\mathrm{t}}$ (solid line in Fig. 2a). The drop of the current $I_{\mathrm{t}}$ can be calculated

$$
\mathrm{NDR}=\frac{I_{\mathrm{t}}^{\mathrm{I}}-I_{\mathrm{t}}^{\mathrm{II}}}{I_{\mathrm{t}}^{\mathrm{II}}}=\frac{\gamma_{\mathrm{b} 1} \gamma_{\mathrm{t} 2}}{\left(\gamma_{\mathrm{t} 1}+\gamma_{\mathrm{t} 2}\right) \gamma_{\mathrm{b} 2}},
$$

where $I_{\mathrm{t}}^{\mathrm{I}} \mathrm{i} I_{\mathrm{t}}^{\mathrm{II}}$ denotes the currents on the first (I) and the second (II) plateau, respectively (see Fig. $2 \mathrm{a}$ ). When $\gamma_{\mathrm{b} 2} \ll \gamma_{\mathrm{b} 1}$, then the charge accumulation on the bottom island is large. It leads to large NDR (Eq. (3)).

The results presented in Fig. 2 were calculated in a particular case when the bias voltage in the top SET is equal to the bias voltage in the bottom one. The results in a more general case are plotted in Fig. 3 . There is the contour plot of the $I_{\mathrm{t}}$ as a function of $V$ and $V_{2}$. From Fig. 3 one can determine for which voltages the negative differential resistance effect appears. If the relation $V_{2}=1.75 \mathrm{~V}$ is fulfilled (if we are going from point $(0,0)$ along dashed line), the NDR effect occurs twice. 
The effects can be use to built three-state switches.

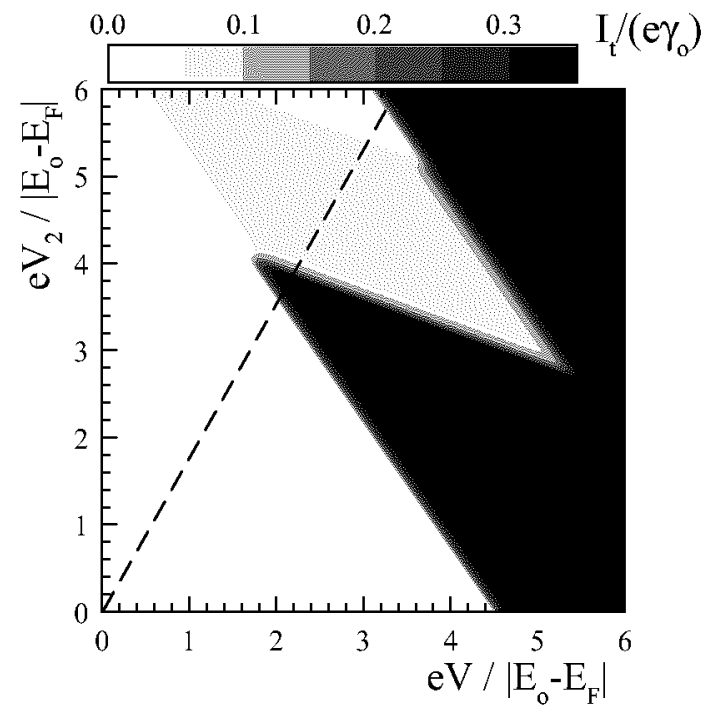

Fig. 3. The contour plot of the current $I_{\mathrm{t}}$ flowing through the top SET as a function of $V$ and $V_{2}$. The parameters are the same as in Fig. 2.
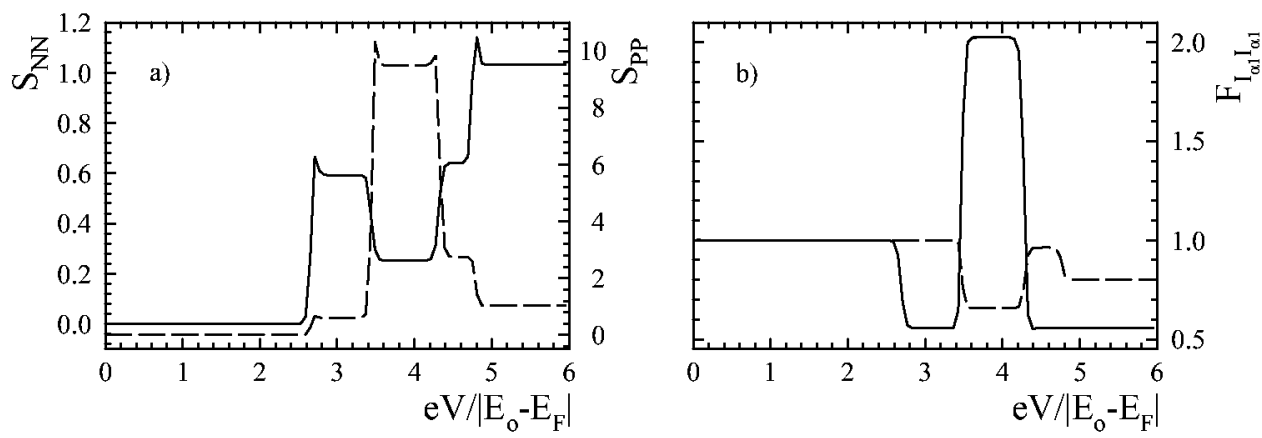

Fig. 4. (a) Total charge $-S_{\mathrm{NN}}$ (solid line) and polarization $-S_{P P}$ (dashed line) noises. (b) Fano factor of the current noise in the top (solid line) and the bottom (dashed line) transistor. The parameters are the same as in Fig. 2.

To characterize dynamics of the system we have calculated the dependence of the charge and the current noise versus the bias voltage and the frequency. The numerical results are presented in Fig. 4 a for the total charge noise $S_{N N}$ (for $N=N_{\mathrm{t}}+N_{\mathrm{b}}$ ) and the polarization noise $S_{P P}$ (for $P=N_{\mathrm{t}}-N_{\mathrm{b}}$ ) versus $V$ at the frequency $\omega=0$. The functions of $S_{N N}$ and $S_{P P}$ are step-like. Opening of a new charge channel leads to changes of the shot noises. The increase in the $S_{N N}$ corresponds to the increase in the charge accumulation on the top islands (compare Fig. 2 and Fig. 4). In the NDR (and in the second plateau II) region (for 
the top transistor) one can see an enhancement of the polarization noise $S_{P P}$. The amplitude of $S_{P P}$ is one order of magnitude larger than the charge noise $S_{N N}$.

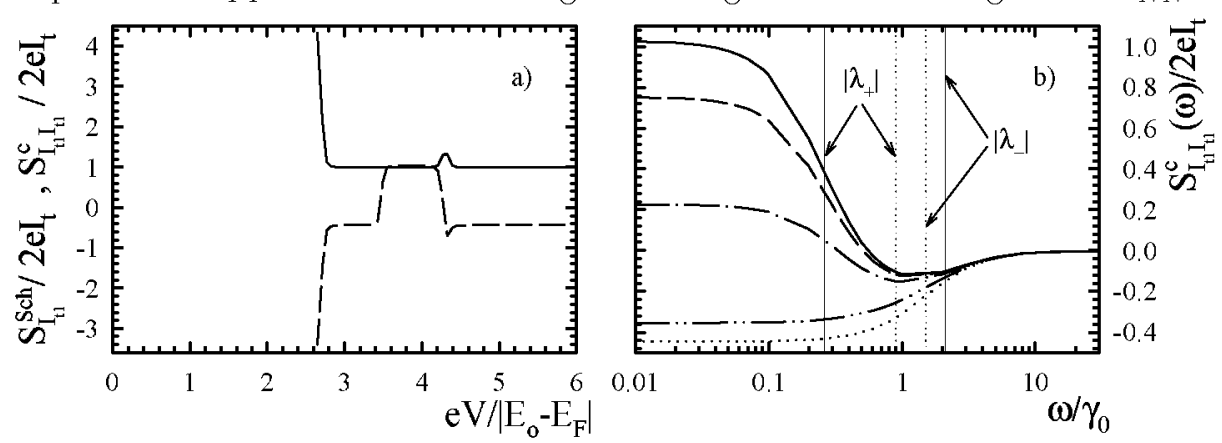

Fig. 5. (a) Decomposition of the Fano factors on the Schottky noise (solid line) and the frequency dependent part (dashed line). (b) Power spectrum of the frequency dependent part of the current noise $S_{I_{11} I_{11}}^{c}(\omega)$ for the voltages $V=3.0$ (dotted line), $V=3.43$ (dash dot dot line), $V=3.47$ (dash dot line), $V=3.50$ (dashed line) and $V=3.8$ (solid line). Thin vertical lines denote values of $\lambda_{ \pm}$for the voltages $V=3.0$ (dotted line) and $V=3.8$ (solid line). The parameters are the same as in Fig. 2.

The current noise can be written as the sum of the coefficient, which is frequency independent (the Schottky noise) and a frequency dependent part

$$
S_{I_{\mathrm{t} 1} I_{\mathrm{t} 1}}(\omega)=S_{I_{\mathrm{t} 1}}^{\mathrm{Sch}}+S_{I_{\mathrm{t} 1} I_{\mathrm{t} 1}}^{\mathrm{c}}(\omega)
$$

The results of the calculation of the Fano factor $F_{I_{\mathrm{t} 1} I_{\mathrm{t} 1}}=S_{I_{\mathrm{t} 1} I_{\mathrm{t} 1}}(0) / 2 e I_{\mathrm{t}}$ are shown in Fig. 4b. In general, the current shot noise is suppressed below the Poissonian value due to the negative correlation between tunneling electrons. However, in the region of the second plateau (i.e. for $V=3.8\left|E_{0}-E_{\mathrm{F}}\right| / e$ ) the shot noise is super-Poissonian. Decomposition of the current noise into two parts is presented in Fig. 5a. The enhancement of the Schottky noise at the voltage threshold is due to activation of the tunneling processes through the first junction at the top SET. The frequency dependent part (for $\omega=0$ ) has negative values due to the Coulomb blockade effect. Because the Schottky noise is voltage independent (excluding the voltage region at the $\left.V_{\mathrm{t}}{ }^{\mathrm{th}}\right), S_{I_{\mathrm{t}} I_{\mathrm{i} 1}}^{\mathrm{c}}$ is responsible for the enhancement of the current noise in the region of the second plateau (Fig. 5a).

For the voltage $V=3.8\left|E_{0}-E_{\mathrm{F}}\right| / e$, we found analytical formulas for the Fano factors. After the spectral decomposition the frequency dependent part of the current noise has a form

$$
S_{I_{\alpha i} I_{\beta j}}^{c}(\omega)=\frac{2 e I_{\alpha}}{\lambda_{-}^{2}-\lambda_{+}^{2}} \sum_{r= \pm} r \frac{a_{\alpha i, \beta j}+b_{\alpha i, \beta j} \lambda_{r}^{2}}{\omega^{2}+\lambda_{r}^{2}}
$$

where $a_{\alpha i, \beta j}$ and $b_{\alpha i, \beta j}$ depend only on the tunneling rates $\gamma_{\alpha i}, \lambda_{ \pm}$are the eigenvalues of the matrix $\widehat{M}$. 
Let us analyze the current noise at the top SET through the first junction. At $\omega=0$ one can write

$$
S_{I_{\mathrm{t} 1} I_{\mathrm{t} 1}}^{\mathrm{c}}(0)=2 e I_{\mathrm{t}} \frac{a_{\mathrm{t} 1, \mathrm{t} 1}}{\lambda_{-}^{2} \lambda_{+}^{2}},
$$

where $a_{\mathrm{t} 1, \mathrm{t} 1}=-2 \gamma_{\mathrm{t} 1} \gamma_{\mathrm{t} 2}\left(\gamma_{\mathrm{b} 2}^{2}+\gamma_{\mathrm{b} 1} \gamma_{\mathrm{b} 2}-\gamma_{\mathrm{t} 2} \gamma_{\mathrm{b} 1}\right)$. It is seen that the current noise is super-Poissonian for $a_{\mathrm{t} 1, \mathrm{t} 1}>0$, i.e. when $\gamma_{\mathrm{t} 2}>\gamma_{\mathrm{b} 2}+\gamma_{\mathrm{b} 2}^{2} / \gamma_{\mathrm{b} 1}$. On the other hand, the noise is suppressed below the Poissonian value even in the NDR region. We selected some voltages to illustrate the change of the frequency dependent shot noise while we are going from the first to the second plateau, see Fig. 5b. One can see that the power spectrum of the current noise can be either positive or negative. For the voltages $V=3.43,3.47,3.5,3.8$ in units $\left|E_{0}-E_{\mathrm{F}}\right| / e$ the current-current correlation function is positive in a low-frequency regime, while for a higher frequency can be negative. The curves (Fig. 5b) are the sum of the two Lorentzian lines with different relaxation times $\tau_{+}=-1 / \lambda_{+}$and $\tau_{-}=-1 / \lambda_{-}$, respectively. We have found that the charge noise $S_{N N}$ disappears with the characteristic relaxation time $\tau_{-}$corresponding to the fast charge fluctuations, while $\tau_{+}$characterizes the polarization fluctuations. From Fig. $5 \mathrm{~b}$ one can see that low-frequency processes are responsible for the enhancement of the current noise. We can say that current shot noise is super-Poissonian due to activation of the fluctuations of the polarization.

In this paper we have calculated the transport properties in the steady state and analyzed the shot noise in the system with two SETs connected in parallel. We have found that the charge accumulation is responsible for the NDR phenomenon. The spectral analysis of the current-current correlation function shows that an enhancement of the current noise is due to the activation of the polarization noise.

The work in this paper was supported by the State Committee for Scientific Research under grants No. 2 P03B 14019 (G.M.) and No. 2 P03B 08719 (B.R.B.).

\section{References}

[1] C.P. Heij, D.C. Dixon, P. Hadley, J.E. Moij, Appl. Phys. Lett. 74, 1042 (1999).

[2] J. Chen, M.A. Reed, A.M. Rawlett, J.M. Tour, Science 286, 1550 (1999).

[3] H. Birk, M.J.M. de Jong, C. Schönenberger, Phys. Rev. Lett. 75, 1610 (1995).

[4] G. Iannaccone, G. Lombardi, M. Macucci, B. Pellegrini, Phys. Rev. Lett. 80, 1054 (1998).

[5] Quantum Transport and Dissipation, Eds. T. Dittrich, P. Hänggi, G.-L. Ingold, B. Kramer, G. Schön, W. Zwerger, Wiley-VCH Verlag, New York 1998.

[6] K.M. van Vliet, J.R. Fassett, in: Fluctuation Phenomena in Solids, Ed. R.E. Burgess, Academic Press, New York 1965, p. 267.

[7] A.N. Korotkov, Phys. Rev. B 49, 10381 (1994); S. Hershfield, J.H. Davies, P. Hyldgaard, C.J. Stanton, J.W. Wilkins, Phys. Rev. B 47, 1967 (1993). 